\title{
Physics students' construction and checking of differential volume elements in an unconventional spherical coordinate system
}

\author{
Benjamin P. Schermerhorn ${ }^{1}$ and John R. Thompson ${ }^{1,2}$ \\ ${ }^{1}$ Department of Physics and Astronomy, University of Maine, Orono, Maine 04469, USA \\ ${ }^{2}$ Maine Center for Research in STEM Education, University of Maine, Orono, Maine 04469, USA
}

(Received 25 May 2018; published 14 February 2019)

\begin{abstract}
In upper-division physics courses, students' use of differential line, area, and volume elements and their facility with the various multivariable coordinate systems consistently go hand in hand. As part of an effort to investigate student understanding of the structure of non-Cartesian coordinate systems and the associated differential elements, we interviewed students (mostly in pairs) in junior-level electricity and magnetism courses at two universities. In a sequence of tasks, students were asked to construct a differential length vector and a differential volume element in an unconventional spherical coordinate system. None of the students were able to arrive at a correct differential length element initially. This work addresses the construction and checking of the volume element. Volume element construction occurred by either combining associated lengths, an attempt to determine sides of a differential cube, or mapping from the existing spherical coordinate system. Students who constructed volume elements from differential length components corrected their length element terms as a result of checking the volume element expression by integration. Other students who relied heavily on spherical coordinates displayed further difficulty connecting dimensionality and projection ideas to differential construction.
\end{abstract}

DOI: 10.1103/PhysRevPhysEducRes.15.010112

\section{INTRODUCTION}

An understanding of mathematical concepts, equations, and expressions is often key to the foundational understanding of upper-division physics. Research on student learning in electricity and magnetism $(\mathrm{E} \& \mathrm{M})$ has indicated several categories of difficulty related to mathematics, including accounting for underlying physical symmetry, extracting information from physical situations for calculation, and interpreting the results of calculation physically [1]. Vector calculus, including vector integration and vector differential operators, is ubiquitous across the E\&M curriculum, often providing the underlying representation for relationships between various concepts. A crucial aspect of problem solving in E\&M is setting up the mathematical expressions for desired quantities, often in integral or differential form, based on the physical scenario. The prominent role of multivariable calculus operators requires students to have a reasonable command of differential quantities in a two- or three-dimensional space. Additionally, due to the high instantiation of nonCartesian symmetry, understanding of these differential

Published by the American Physical Society under the terms of the Creative Commons Attribution 4.0 International license. Further distribution of this work must maintain attribution to the author(s) and the published article's title, journal citation, and DOI. quantities is often mitigated by an understanding of spherical or cylindrical coordinate systems and the associated differential length, area, and volume elements.

The variation in the use of coordinate systems is one of the key factors in the "vector-calculus gap," [2-4] which represents the pedagogical and conceptual differences between the two disciplines of mathematics and physics. Among the differences is the idea that mathematics courses predominantly use Cartesian coordinates, whereas physicists often choose a coordinate system from the symmetry of the physical scenario. Other work in this area notes a large concern over the lack of standardization of variable labeling conventions in non-Cartesian coordinates between disciplines [2]. For this work, we will use the physics convention for spherical coordinates, which labels the azimuthal angle as $\theta$ and the polar angle as $\phi$.

Beyond this, a survey of calculus curricula reveals different methods of integration over volumes [5]. Volume integration in mathematics is typically approached by thinking about the area between two functions and finding the volume obtained by rotating that area about a specific axis, or by finding the volume enclosed between two planar surfaces. In E\&M, volume integration is commonly used to determine the total charge of a given object (e.g., sphere or cylinder) with a given charge distribution. In these tasks, students are expected to integrate the product of the charge density and a differential volume element expressed in the appropriate coordinate 
system. As many of the physical scenarios in E\&M are most easily solved in a non-Cartesian system, differential volumes include scaling factors that account for the curving of spherical $\left(d \tau=r^{2} \sin \theta d r d \theta d \phi\right)$ or cylindrical $(d \tau=s d s d \phi d z)$ space, rather than the straightforward $d x d y d z$ from a rectangular coordinate system.

While scaling factors can be determined through a Jacobian or coordinate transformation, they can also be constructed less formally with an understanding of the underlying geometry. The latter involves recognizing that the curvature of the space necessitates arc lengths to represent some of differential length components and that the resultant volume element is composed of a product of the magnitude of the length components. The length component and subsequent volume component for spherical coordinates are given by

$$
\begin{aligned}
\overrightarrow{d l} & =d r \hat{\mathbf{r}}+r d \theta \hat{\boldsymbol{\theta}}+r \sin \theta d \phi \hat{\boldsymbol{\phi}}, \\
d \tau & =(d r \hat{\mathbf{r}}) \cdot(r d \theta \hat{\boldsymbol{\theta}} \times r \sin \theta d \phi \hat{\boldsymbol{\phi}}) \\
& =(d r)(r d \theta)(r \sin \theta d \phi)=r^{2} \sin \theta d r d \theta d \phi .
\end{aligned}
$$

However, as shown in the final form of the volume element above, most conventions for writing the differential volume element involve the scaling factors written out by variable in front of the set of differentials, obscuring the origin of the term as a product of differential lengths.

Previous research has addressed student use and understanding of many aspects of vector calculus quantities in the context of E\&M, including differential elements [6], integration [7-10], applications of symmetries for Gauss's law and Ampère's law [1,11-15], and vector differential equations in mathematics and physics settings [16,17]. But despite the centrality and ubiquity of non-Cartesian symmetry in E\&M problems requiring vector calculus operations, little attention has been given to student understanding of differential elements in non-Cartesian coordinate systems, and the extent to which these elements are used in a rote fashion or whether the structure of the expressions has meaning to students when employed. As part of a broader study to investigate these issues, we developed an interview task in which students were asked to construct a differential length vector and a differential volume element for a spherical coordinate system where variable labels and placement are changed from standard conventions. Findings from the differential length construction part of the task are presented in a companion paper [18]. The results presented here address students' construction of differential volume elements, in line with the following research questions:

(1) To what extent do students understand the multivariable coordinate systems used in E\&M?

(2) What ways do students build and/or determine differential volume elements in multivariable systems?
(3) To what extent do students connect a differential volume element to the differential length components in a given non-Cartesian coordinate system?

Addressing these questions provides knowledge of students' geometric understanding of non-Cartesian coordinate systems, as well as insight into how understanding of the differential elements used in may E\&M expressions.

\section{CONTEXT FOR RESEARCH}

Course observations were conducted in the first semester of junior-level E\&M at the first of two universities (university A). These observations revealed discrepancies in students' performance when writing differential elements for spherical and cylindrical coordinate systems. It is in this course that students first encountered these multivariable coordinate systems and differential vector elements. Spherical coordinates were introduced and used for several units of class before the introduction of cylindrical coordinates. Once both coordinate systems had been introduced and used in standard examples, an in-class quiz was administered as part of regular instruction. Results showed more students were able to construct differential length vectors in cylindrical coordinates; as the course progressed, homework and exam data suggested students were more proficient with spherical differential elements when solving various integration tasks. This suggested underlying difficulties in students' understanding of how differential elements are constructed and used in particular coordinate systems, and suggested that performance on spherical coordinates was due to extended use early in the semester.

These observations prompted investigations into students' conceptual and symbolic understanding of differential elements in non-Cartesian coordinate systems within and without physics context [18,19]. As reported in a companion article, analysis of differential length construction showed student attention to various conceptual aspects and symbolic structures needed to express a threedimensional differential length vector [18]. However, no student was initially able to completely construct a correct length element. This work explores students' construction of the differential volume element for the unfamiliar system as well as students' checking of the correctness of the element in terms of the ideas accessed during the initial length construction, as well as the connections made between the differential length vector and differential volume for a given coordinate system.

\section{RELATED LITERATURE}

Research on student understanding of vector calculus in E\&M has addressed topics in several key areas. Much of this work has explored student understanding of Gauss's and Ampère's laws, expressed as a flux and line integral, respectively. These laws are frequently employed in E\&M in the abundance of highly symmetric cases. Thus, much of the 
literature in either case focuses on students' recognition and/or application of symmetry. It is common for students to overgeneralize the use of either law to include cases where symmetry is not present, or attempt to apply any given coordinate symmetry as long as the Gaussian surface or Ampèrian loop encloses the desired charge or current [1,11-15].

Other work within the realm of vector calculus has explored student understanding of vector differential operators and students' interpretations of vector fields $[16,17]$. Researchers found students were adept at procedural calculation when provided tasks involving gradient, divergence, and curl, but were unable to appropriately express the conceptual meaning of the operations [20]. These difficulties speak to the larger difficulties that students have with the application and interpretation of mathematics at this level, as categorized by Pepper and colleagues: assessing underlying physical symmetry, establishing mathematical representations of physical situations for the purpose of calculation, and interpreting the results of calculation in terms of the given physical situation [1].

Pepper and colleagues briefly noted two cases of student difficulties with construction of differential elements. In one case, students neglected to include the necessary scaling factors when writing spherical differential areas, using $d a=d \theta d \phi$, rather than $d a=r^{2} \sin \theta d \theta d \phi$. This is reminiscent of students' attempts to pattern match a product of two differentials in a non-Cartesian system with one in Cartesian coordinates [21,22]. Research has shown students at various levels are less comfortable when working within polar coordinates [22-25]. In a second example, a group attempted a three-dimensional line integral using $d x d y d z$ as a path length element. These types of errors show difficulty with understanding and constructing differential elements in multivariable coordinates that have been relatively unexplored before now.

Student understanding of calculus concepts has been another area of focus in E\&M. Hu and Rebello have investigated student understanding of differentials in the context of integration of charge or resistivity along one dimension [6]. Several resources and conceptual metaphors were used by students across these tasks, establishing four common treatments of differential quantities: derivatives as small amounts, as unitless points, as a cue to differentiate a formula to derive a second differential quantity, and as an indicator of the variable of integration. The identification of the differential as a small amount can be connected to a specific cue for students to integrate, where students identify the need to add up "little chunks" using an integral $[7,8]$. However, research in mathematics education has commonly reported student treatment of the differential as a meaningless quantity that only serves to identify the variable of integration [26-29]. The sum of this work highlights the fact that many students do not connect the differential quantity to a physical meaning, even when given a specific context. While addressing larger concerns about students' treatment of integration and differentials, these studies largely focused on integration in one dimension, or on quantities such as resistance or capacitance.

Therefore, despite significant forays into various levels of mathematical understanding, little work has explored student understanding of the differential vector element, in particular as expressed in the non-Cartesian coordinate systems used in physics problems. This work takes a reasonable next step toward analysis of student understanding of one of these elements - the differential volume element - as it appears in non-Cartesian coordinate systems used in E\&M.

\section{THEORETICAL PERSPECTIVE}

Building largely off of work on student construction of differential length elements within the same task [18], we analyze student construction of differential volume elements using a concept image framework [30] to make explicit connections to earlier work as well as address new ideas related specifically to differential volume elements.

A student's concept image is the multifaceted cognitive structure that includes all the properties, processes, mental pictures, or ideas that they associate with a particular topic. For example, students may have multiple ways to think about integration: with a Riemann sum, area under the curve, or anti-derivative approach. A concept image would additionally contain associated properties for each of these methods. The sum of these ideas that the student associates with integration make up the student's full concept image; however, a specific task or context may only elicit one of these approaches [8]; this is referred to as the evoked concept image for that task or context. While a student may have other ideas related to integration, determining a student's evoked concept image for a particular task (e.g., area under the curve) allows insight into how a student approaches a problem in a given context. Likewise, it is also telling if a student only has a rule-based understanding, e.g., the integral of $n x^{(n-1)} d x$ is $x^{n}+C$, without an understanding of the underlying meaning. The particular concept image evoked for a given topic dictates the approach and properties a student would use for a given task.

Notably, as a student continues to apply and extend an idea, their concept image grows and may pick up ideas that are false or that contradict earlier aspects. In some cases, a student can develop what is referred to as a restricted concept image if the student learns and applies a concept in a very specific way for an extended period of time. Following the development of a restricted concept image, a student may later have difficulty extending the specific conceptual understanding to a broader context. For example, a student learning Coulomb's law who then spends several weeks using Gauss's law may develop a restricted concept image of integration of electric fields, and may attempt to apply Gauss's law in a case where symmetry is absent, a situation 
well documented in the literature [1,11-13]. The formation of a restricted concept image is a reasonable way to describe algorithmic knowledge without conceptual understanding. In these cases, students have only learned a particular concept as a computational entity (e.g., integrals as antiderivatives) and have not been asked to interpret or make sense of the computation. The absence of appropriate ideas or procedures within a student's restricted concept image can contribute to difficulties solving a given task and may further cause the student to rely, correctly or incorrectly, upon the mathematical procedure without deeper physical understanding.

The use of concept image as an analytical perspective has recently been adopted by physics education researchers studying students' mathematical reasoning in the context of vector integration [8] and differential vector operators in electromagnetism courses [17], as well as to identify the specific properties and associations students used (or neglected to use) when constructing the differential length element for an unconventional coordinate [18].

In the context of this work, we use concept image to address the ways in which students think about the differential volume element and to provide further insight into understanding of the properties related to $\overrightarrow{d l}$.

\section{RESEARCH DESIGN AND METHODOLOGY}

In order to investigate student understanding of associated differential elements, a task was developed in which students were asked to construct expressions for differential elements of an unconventional spherical coordinate system that we called "schmerical coordinates" (Fig. 1) [18]. While schmerical coordinates are left handed, the most noticeable difference in the system from spherical coordinates is the placement of the polar angle: while theta ranges from 0 (the $z$ axis) to $\pi$, increasing away from the $z$ axis, the schmerical coordinates polar angle, alpha, increases towards the $z$ axis (up), and ranges from $-\pi / 2$ to $\pi / 2$ (the $z$ axis), with $\alpha=0$ corresponding to the $x y$ plane. This necessitates the use of $\cos (\alpha)$ rather than $\sin (\theta)$ to describe the projection used to construct the azimuthal component of a vector. This change then carries through to the construction of the differential volume element, but becomes abstracted from its origin as a projection. The use of an unconventional coordinate system enabled observation of conceptual exposition in the construction process and reduced the effect of recall of memorized quantities as static knowledge. Interviews used a semistructured protocol with the following series of tasks and questions:

(1) Does this depict a feasible coordinate system and if it is valid, what type of situations (kinds of problems) would it be appropriate for?

(2) Construct a differential length vector $\overrightarrow{d l}$, for the system.

(3) Construct a differential volume element for this coordinate system.

(4) Check that the volume element is correct.

Clinical think-aloud interviews were conducted with students in a junior-level E\&M sequence at two universities. Four pairs of students $(N=8)$ were interviewed at one university (university A) at the end of the first semester of a two-semester sequence. In order to access a larger student population, two pairs and a single student $(N=5)$ were interviewed at a second university (university B) at the beginning of the second semester of this same sequence. Both courses followed a similar structure starting with electrostatics, used the same textbook [31], and were predominately lecture based. However, the course at university A placed specific instructional emphasis on coordinate systems. Students were selected on a volunteer basis and represented a mixture of low to high achievers. Pairs were formed based solely on students' availability. The use of pair interviews facilitated authentic discussion between students where they could arrive at a single answer with minimal input or influence from the interview. Groups are identified as $\mathrm{AB}, \mathrm{CD}$, etc., with individual students given pseudonyms associated with the letters (e.g., Adam and Bart for $\mathrm{AB}$ ).

Interviews were videotaped and fully transcribed. Analysis used open coding to identify common actions and recurring ideas across interview groups with the purpose of identifying the ways in which students constructed and (a)

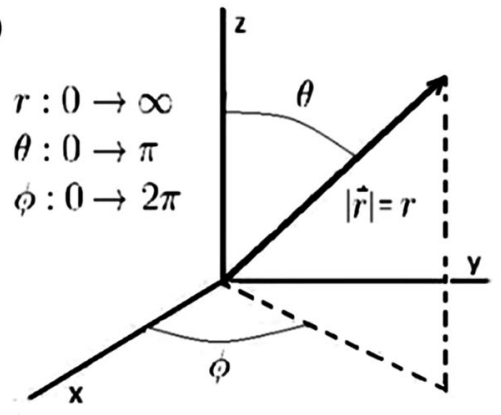

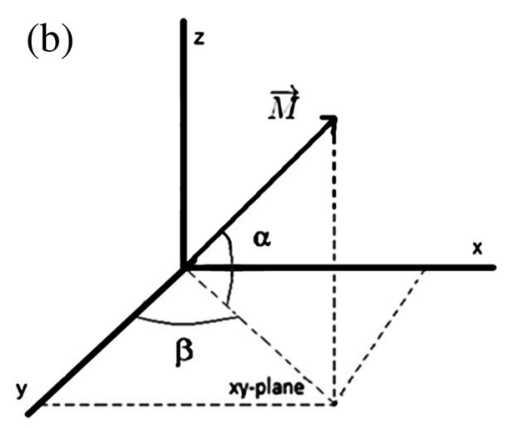

(c) $\overrightarrow{d l}=d r \hat{r}+r d \theta \hat{\theta}+r \sin \theta d \phi \hat{\phi}$ $d \tau=r^{2} \cos \theta d r d \theta d \phi$

(d)

$$
\begin{aligned}
& \overrightarrow{d l}=d M \hat{M}+M d \alpha \hat{\alpha}+M \cos \alpha d \hat{\beta} \\
& d \tau=M^{2} \cos \alpha d M d \alpha d \beta
\end{aligned}
$$

FIG. 1. (a) Conventional (physics) spherical coordinates; (b) an unconventional spherical coordinate system given to students, for which they were to construct differential length and volume elements. The correct elements for each system are in (c) and (d), respectively. 
TABLE I. Aspects of students' concept image of a differential length vector in a non-Cartesian coordinate system [18].

\begin{tabular}{|c|c|c|}
\hline Concept image aspect & Associated idea & Example (in bold) \\
\hline $\begin{array}{l}\text { Component and } \\
\text { direction }\end{array}$ & $\begin{array}{l}\text { Recognition of multiple components, } \\
\text { each displaced independently }\end{array}$ & $\begin{array}{l}\text { Frank: Yeah, so like there, } d l \text {, there are three different } d l \text { 's. There } \\
\text { is } d l \text { with respect to } M, d l \text { with respect to a, } \alpha \text {, and } d l \text { with } \\
\text { respect to } \beta \ldots\end{array}$ \\
\hline Dimensionality & Each term needs units of length & $\begin{array}{l}\text { Adam:... This doesn't have any units of length ... so, it needs to } \\
\text { have some } M \text { term. }\end{array}$ \\
\hline Differential & Small changes (of displacements) & $\begin{array}{l}\text { Carol: Right. So you have a change in your } \hat{\mathbf{M}} \text { is going to be your } \\
d M \text {, it's your change in your } M \text {. }\end{array}$ \\
\hline Projection & $\begin{array}{l}\text { Use of cosine or sine explicitly } \\
\text { (not rote recall) }\end{array}$ & $\begin{array}{l}\text { Elliot: ... but if we're pointed way up here, then we need to take } \\
\text { the cosine so that we're, we have the component of } r \text { that is } \\
\text { actually in the } \beta \text { plane,... }\end{array}$ \\
\hline
\end{tabular}

conceptualized differential volume elements. Categories were coded by the first author, then refined and recoded to the agreement of both authors.

Student responses to the first two questions or tasks, in which students were asked to judge the reasonableness of the coordinate system and to construct a differential length vector, are discussed in a companion paper [18]. That initial analysis categorized these ideas as aspects of students' concept images [30]. Concept image aspects and building actions associated with differential length construction that emerged from this study are reproduced in Tables I and II, respectively. Concept image aspects include component and direction, dimensionality, differential, and projection. Building actions involved recall of and transliteration from other coordinate systems, as well as grouping of specific terms.

The current work addresses student responses to the last two tasks, in which students were asked to construct a differential volume element and subsequently check the correctness of that element. As the differential volume construction task followed the differential length construction task, analysis sought to specifically connect student ideas associated with differential lengths to their construction of volume elements.

\section{RESULTS AND DISCUSSION}

The schmerical coordinates differential volume element task took place after completion of the differential length vector construction task for the system. This task involved asking students to construct a differential volume element and then to check the correctness of the differential volume element.

\section{A. Construction of a schmerical differential volume element}

Groups constructed the schmerical differential volume elements in three distinct ways. Three pairs utilized a concept image of $d \tau$ as the product of length components and easily multiplied the previously determined components. With mixed results, two pairs had previously attempted to monopolize upon this product understanding by recalling a spherical differential volume element and extracting the length components for comparison to their schmerical $d l$ construction. Not having accessed the component and direction concept image aspect of the differential length vector, one group evoked a more pictorial or geometric concept image of the differential volume, and the other group attempted to reconstruct the sides based on coordinate system geometry. The latter approach is considered distinct because while this group connected the volume to the product of lengths, they did not connect the sides of their constructed differential volume to the need for three components of a differential length vector. Lastly, the remaining groups could not exploit the product of length components concept image at all, typically either expressing a length element in Cartesian

TABLE II. Actions taken by students during construction of a differential length vector for schmerical coordinates [18].

\begin{tabular}{|c|c|c|}
\hline Construction action & Associated idea & Example \\
\hline Grouping & Combining elements by like variables or terms & $\begin{array}{l}\text { Harold: You've got } r d r \hat{\mathbf{r}} \text { plus, is it } \sin \theta d \theta \text { or is there an } r \\
\text { in there? }\end{array}$ \\
\hline Rote Recall & $\begin{array}{l}\text { Writing or remembering elements from } \\
\text { Cartesian or spherical coordinate systems }\end{array}$ & Greg: $d \tau$ in spherical is $r^{2} \sin \theta=\ldots=d \theta d r=\ldots=d \phi$ \\
\hline Transliteration & $\begin{array}{l}\text { Direct matching of variables from existing } \\
\text { coordinate system }\end{array}$ & $\begin{array}{l}\text { Bart: ... so now we have just to compare so we have } r \text { it is } \\
\qquad M, \theta \text { is } \alpha=\ldots=\phi \text { is } \beta \text {. }\end{array}$ \\
\hline
\end{tabular}


TABLE III. List of methods used by students to determine the differential volume element and groups having used each method.

\begin{tabular}{lc}
\hline \hline $\begin{array}{l}\text { Method of } d \tau \\
\text { construction }\end{array}$ & Student pairs using this method \\
\hline $\begin{array}{l}\text { Product of length } \\
\text { components }\end{array}$ & $\begin{array}{c}\text { Adam and Bart, Carol and Dan, } \\
\text { Elliot and Frank, Greg and Harold }\end{array}$ \\
$\begin{array}{l}\text { Labeling sides of } \\
\text { differential volume }\end{array}$ & Rachel and Silas \\
$\begin{array}{l}\text { Comparison to spherical } \\
\text { volume element }\end{array}$ & $\begin{array}{c}\text { Correct mapping: Rachel and Silas } \\
\text { Direct transliteration: Greg and }\end{array}$ \\
\hline \hline
\end{tabular}

components or expressing the differential length as a single component in the $\hat{\mathbf{M}}$ direction. They determined $d \tau$ by mapping to the more familiar spherical volume element. These results are summarized in Table III.

\section{Volume as a product of differential length components}

When asked to construct a volume element for schmerical coordinates, $\mathrm{AB}, \mathrm{CD}$, and $\mathrm{EF}$ immediately knew to take a product of differential length magnitudes.

\section{Interviewer: Okay, so can you make a differential volume element?}

Adam: Sure, just multiply them all together.

Each of these groups had constructed a differential length vector with three components based on the unit vectors of the unconventional system [18]. However, due to errors with differential length construction, the constructed differential volumes included an incorrect trigonometric function (Fig. 2), or lacked the trigonometric function entirely.

While the creation of a differential volume as a product may seem trivial, during length construction (the second of four tasks), students having difficulty with direct recall to a spherical $\overrightarrow{d l}$ struggled to isolate the length components from the more easily recalled spherical volume. For example, after recalling the spherical differential volume expression, Carol explicitly recognized that the differential volume element is constructed from a product of length components and that the terms are grouped differently in the volume element.

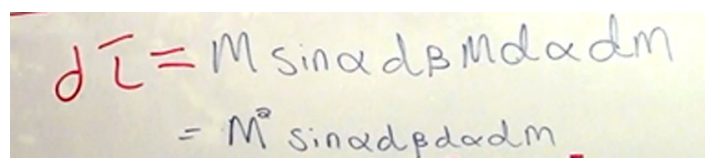

FIG. 2. Final differential volume constructed by Carol and Dan including incorrect trigonometric function.
Carol: ... I was trying to figure out which, I guess, um, I don't know, vector direction each come from, um, because I feel like, right? This is right, right? We just write it $r^{2}$ for convenience, right? It comes from separated out [terms].

Carol and Dan then began to check the units (dimensionality) of terms to check their separation of components. Similarly, Greg and Harold recall the spherical $d \tau$ in an attempt to recall the spherical length vector.

However, rather than recreating the appropriate length components, both pairs grouped angular terms based on variables (Fig. 3), pairing the $\sin \theta$ with the $d \theta$ similar to how the terms would appear in multivariable integration. Because this is what the differentials are typically used for in solving E\&M problems, the typical expressions for differential volume elements (e.g., $r^{2} \sin \theta d r d \theta d \phi$ for spherical coordinates) involve a grouping of terms in a way that dissociates the variables from their particular length component. Students' coupling of the theta terms and ease of recalling the spherical volume element over the assembly of the volume element from the differential length components supports the idea that students do not have the fundamental understanding of non-Cartesian systems necessary for interpreting vector calculus in $\mathrm{E} \& \mathrm{M}$.

After some time, Carol and Dan were able to correct the grouping error, when Dan made the explicit connection to length vector construction in spherical coordinates and connected the $\sin \theta$ to a projection into the plane of the polar angle [18]. Because of transliteration of terms, this lead to a $\sin \alpha$ in their length component that carried over into their $d \tau$ as they multiplied length terms (Fig. 2).

However, for Greg and Harold, the dissociation from length components was much more complicated, as neither student attended to the necessary dimensionality [Fig. 3(b)].

(a)

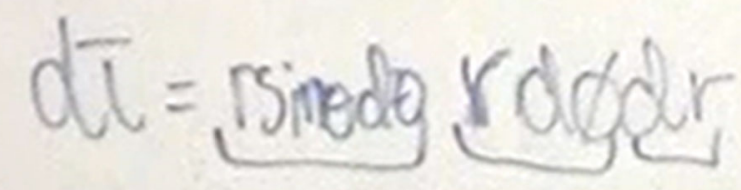

(b)

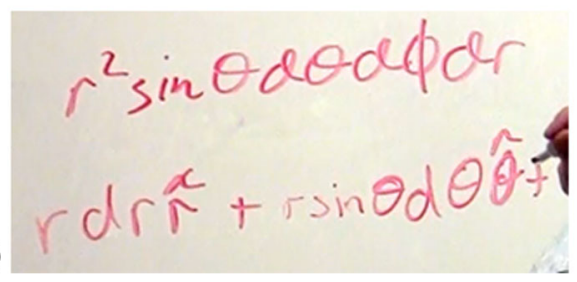

FIG. 3. (a) Incorrectly distributed length terms in a spherical differential volume written by Carol and Dan. (b) Unsuccessful attempt to reconstruct differential spherical length element by Greg and Harold. 
Harold: You've got rdr $\hat{\mathbf{r}}$ plus, is it $\sin \theta d \theta$ or is there an $r$ in there?

Greg: I think there is an $r$ there. It's an $r$ because you want, you want at that radius uh, plus a small angle.

Harold seemed to have a concept image in which the grouping of terms based on like variables dwarfs the grouping based on correct ideas for each length component. If he had only been concerned with the grouping of variables, all the $r$ terms in the differential length component would have been grouped with $\hat{\mathbf{r}}$. As they decomposed the volume element, they ran out of components to be able to express the remaining $\phi$ component. The pair then abandoned this method of construction and began to express the differential length in terms of Cartesian unit vectors [18]. This goes further to show how a lack of reasoning about dimensionality can hamper problem solving in $\mathrm{E} \& \mathrm{M}$.

\section{Volume as product of sides of a differential cube}

Rachel and Silas entered the volume construction phase of the task after first constructing a differential length vector as a single component accounting only for change in the radial direction. Without the three components, which pairs $\mathrm{AB}, \mathrm{CD}$, and $\mathrm{EF}$ relied upon, Rachel and Silas started their volume construction attempt by drawing a small volume at the end of $\vec{M}$ [Fig. 4(a)]. This construction elicited a discussion of arc length to account for the sides of the volume element, but did not cause the students to reflect upon the single-component differential length vector constructed in the earlier phase of the task.

Rachel: That is like the differential volume element right here, with $d M$ as the thickness. So if alpha changes you have this arc length.

This shows that students' difficulties with length construction may not have been due to lacking the prerequisite ideas, but to having a restricted concept image of the differential length vector as a whole. Given that the majority of problem solving in the electrostatics portion of E\&M involves calculating a change in potential over a

(a)

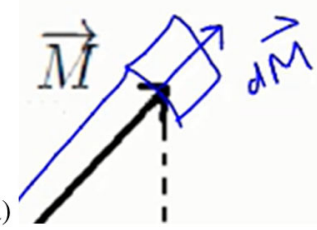

(b)

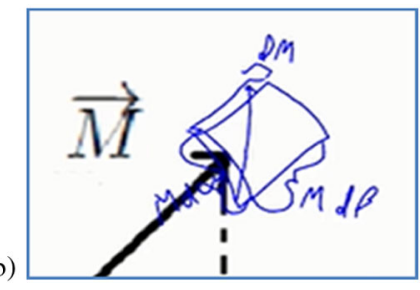

FIG. 4. Physical construction of a differential volume element by Rachel and Silas. (a) Beginning of volume construction using the single length component. (b) Final differential volume, where location of $d M$ has changed. Students do not connect the sides of the volume to length components. radial field, the predominance of such problems early in E\&M may restrict students' concept image to only needing to account for the radial component of the differential.

Yet the ideas of dimensionality and arc length-ideas that other groups correctly attributed to the length component-were elicited from Rachel and Silas once they were able to build the differential volume geometrically. As RS continued in their construction, they correctly represented $M d \alpha$ as the side resulting from a small change in alpha, but placed $M d \beta$ where $d M$ had previously been on their diagram. As a result, $d M$ took on the role of the "thickness" into and out of the page rather than in the radial direction, as previously depicted [Fig. 4(b)]. This highlights a difficulty of visualizing the geometric directions of the schmerical unit vectors. This difficulty could be connected to a student difficulty reasoning about three-dimensional objects within a two-dimensional space, something sparsely studied in mathematics education research $[32,33]$. At the end of this differential volume construction, Rachel and Silas were unsatisfied with their differential volume lacking a trigonometric function, and began to build a volume by making a comparison of variables (mapping) to spherical coordinates.

\section{Volume as mapping from spherical coordinates}

Students who had difficulty with length construction, either constructing a differential length vector with one component (RS, T) or without scaling factors (RS), or who represented the differential length vector in terms of Cartesian symmetry (GH, PQ), could not draw on the same product of terms of the first three groups.

Rather than finding a solution pattern to determine the volume element in schmerical coordinates, students recalled the spherical volume element and then mapped the schmerical variables to the spherical terms. This problem-solving approach is consistent with the transliteration to mathematics epistemic game [34]: students identified the task target quantity, found a related solution pattern, mapped new quantities into the related solution, and ended by evaluating the mapping.

After attempting to construct a physical volume and expressing a need to include trigonometric function in their schmerical differential volume, RS began to match variables to the spherical coordinate system (Fig. 5). Here they appropriately accounted for the relationship between theta and alpha, as $\alpha=-\theta+\pi / 2$. The pair then connected the differentials and rewrote the spherical volume in terms of the associated schmerical variables. They recognized mathematically that the $\pi / 2$ shift of alpha from the original theta turns the $\sin \theta$ to $\cos \alpha$, but they did not connect the change or original trigonometric function to the physical justification of projection.

Rachel: Well okay, so if we have it down in this plane then wait, set alpha equal to 0 right? So it's down in 


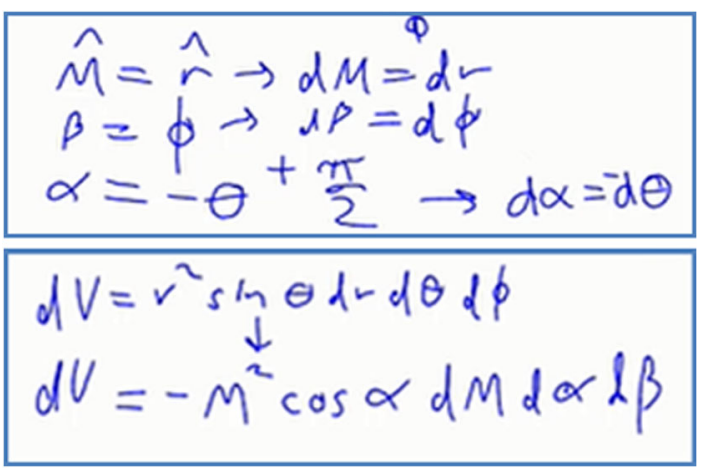

FIG. 5. Rachel and Silas directly compare coordinate systems and account for changes in variable placement as they construct a volume element.

[the xy] plane. I can convince myself that this is cosine. No, no, that's beta. Hold on. I don't even know. Silas: Well I know that is right. I know that much. Rachel: Yeah, ... I just don't know why it is right.

Here Rachel and Silas are able to arrive at the correct expression for the differential volume element by a change in variable but do not recognize that the trigonometric function scales the specific arc length of the $\beta$ component. Without being able to physically justify the cosine, their epistemological stance is to trust the mathematics [35]. This lack of understanding of the reason behind the projection is pervasive across all groups, especially during length construction [18].

After arriving at a correct volume element, RS returned to their differential length vector, but again, due to the lack of a trigonometric function in the drawn volume element, they did not connect the length and volume differential expressions. Rachel and Silas then augmented their length vector to include a $d \alpha$ and $d \beta$, in their respective directions, but failed to recognize the need for arc length discussed previously during the construction of the volume [18]. Additionally they did seek to reconcile the differences between the differential elements as the previous groups did during the checking phase of the task.

Individual subject Tyler and group PQ also attempted to map onto a spherical differential element but did so unsuccessfully, connecting the physics variation of the differential element with the mathematical conventions for the spherical coordinate system $(\theta$ as polar angle, $\phi$ as azimuthal). Compounded with the missing idea of projection in the polar length component, this resulted in differential volume elements that include a $\sin \beta$ instead of a $\cos \alpha$ (Fig. 6).

Having had particular trouble with construction of a differential length vector, Greg and Harold quickly constructed their new $d \tau$ from a direct mapping of the previously recalled spherical differential element. Greg initially accounted for the different placement of alpha by writing $(\pi / 2 \alpha)$ as the argument of the sine function,

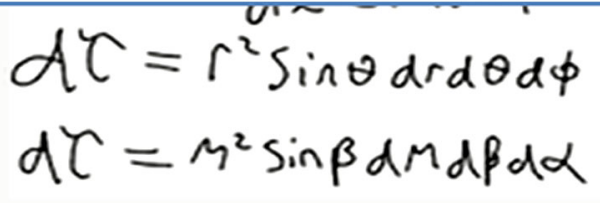

FIG. 6. Tyler's determination of a schmerical differential volume by comparison to spherical. Tyler unknowingly uses mathematics conventions for spherical coordinates.

but then decided a direct replacement of the variable would be sufficient.

Greg: Actually, if you just said $\sin \alpha$ I think it would work. You would just have to know that it points in a different direction.

At this point they returned to the differential length element upon request of the interviewer and eventually reconstructed a correct differential length element based on the process in the course text [31]. When asked if they were still satisfied with their differential volume element, they had difficulty recognizing the need to reconcile the cosine in their length vector with the sine in their volume element.
Harold: I still like our volume element=
Greg: Yeah, I think so.
Harold: = I don't know about you, this one over here,
I still think that.
Greg: They're the same, yeah.
Interviewer: Okay, and can you check that that volume element is correct?
Greg: Isn't that kind of the same question?
Harold: Oh, you want us to actually do this integral out.
Greg: Oh. No, but see in down here we've gone with the $\cos \alpha$.
Harold: Oh, we've gone cosine, oh yeah.
Greg: And so we might want cosine. Yeah, I think we do.
Oh wait, let's see. Oh not, that's, alright. Yeah, we do want these. We want these to agree so they need to be, this needs to be a cosine [in the volume element].

Despite GH's attempt to deconstruct the volume element as a product of terms, their hesitancy to connect the length and volume terms, coupled with the difficulty deconstructing the volume element due to misuse of the grouping of terms and inattention to dimensionality, show that Greg and Harold did not have a strong understanding of the structure of these differential elements.

\section{B. Checking of the schmerical differential volume}

The last phase of the task involved the checking of the differential volume element. This most often involved integration to obtain the expression for the volume of a sphere of constant radius, but in some cases additionally 
involved a dimensional analysis. Students were asked to check their differential volume element if they used terms associated with their (incorrect) differential length vector or mapped incorrectly from spherical coordinates and thus had an incorrect term within their differential volume. Students who mapped correctly were not asked to check their differential volume, as the connection between their differential volume and length elements was weaker and a correct differential volume would not likely lead towards reconciliation between the terms.

For students with differential length elements in which only the trigonometric function was missing or incorrect, the checking of the differential volume elements led to the eventual correction of the differential length vector and solidification of the connection between the trigonometric function and the projection aspect. Groups using recall and transliteration to construct the differential volume element were still not able to recognize the need to invoke projection: the use of cosine remained a mathematical transformation rather than acquiring a geometric justification.

\section{Checking of volumes constructed by a product understanding}

Upon checking their differential volume elements, both $\mathrm{AB}$ and $\mathrm{CD}$ easily recognized that integration of their differential volume would give the expression for the volume of a sphere of radius $M$, but due to their incorrect trigonometric function, integration over the bounds of $\alpha$ yielded a volume of 0 for both groups. This alerted the groups to an error in their length components, which they (quickly) traced to the $\sin \alpha$ term. Adam immediately recognized the mistaken projection that resulted from directly substituting $\alpha$ for $\theta$ during their mapping. He articulated that the change in the placement of the angle meant a $\cos \alpha$ was needed to get the appropriate length component. Carol and Dan were able to recognize that $\sin \alpha$ was the cause of their unexpected result, but did not immediately connect this to the idea of projection.

Carol: 0, which means our volume is wrong. Which means, should this be cosine? No, we need...

Dan: I mean, well our trig might be wrong but we also could be running into the problem that we were incorrect about. Oh... when you assumed $\sin \alpha$, you assumed you were basing it off $\sin \theta$ where $\theta$ was on a different part of the graph.

Carol first suggested cosine as a way to make the mathematics work. It is not until after a couple of incomplete exchanges that Dan connected the mathematical implications of change in trigonometry to the physical difference in the geometry of schmerical coordinates. The construction and checking of the volume component cued projection, the absence of which had previously led to a shift to recall during length construction.

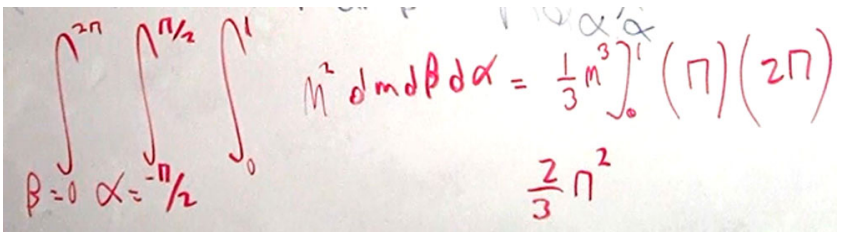

FIG. 7. Elliot and Frank check their incorrect differential volume element by integrating.

When asked to check the volume element, Frank reasoned using dimensionality, saying that integration of the $M$ terms would give units of length cubed and therefore it did not matter what the remaining integrals gave as a result. Unconvinced, Elliot suggested integration of the full differential volume element $M^{2} d M d \beta d \alpha$. As their expression contained no trigonometric function, their integration yielded a result with $\pi^{2}$ in their answer (Fig. 7).

Frank: $\pi^{2}$, so ...

Elliot: We needed that sine in there.

Frank: We need a sine or a cosine so we can get rid of $a \pi$.

Elliot: But I don't know where it comes from... [audible gasp] Oh, I remember where it comes from... like if $r$ is pointing way up here, then we need to get the component that's in the flat plane and then that is times $d \beta$.

The pair recognized they need a trigonometric function to get the appropriate mathematical result, but as with their difficulty during length construction, they could not figure out the particular reason for the inclusion of the term. Shortly after this discussion, Elliot recognized the need for a cosine function to account for the necessary projection and the group corrected their length vector. Just as with CD, EF recognized the mathematical need for cosine but was not immediately able to connect it to the radius term in the $\hat{\boldsymbol{\beta}}$ component.

\section{Checking of volumes constructed by recall and transliteration}

As the pair GH checked their differential volume element, Greg became unsure about the reason for the cosine term, despite earlier work during their second attempt at length construction.

Greg: Why did we change it to cosine?

Harold: I'm sorry?

Greg: Actually wait, no, because the negative sign, the negative $\sin (\pi / 2)$ is one.

This further suggests that projection is not strongly tied to this pair's understanding of the differential elements here. It was upon seeing that the computation resulted in the expected answer that Greg regained comfort with the use of the cosine function. 
The result of Tyler's checking of the volume element, $d \tau=M^{2} \sin \beta d M d \beta d \alpha$, via integration yielded 0 , but he was unable to connect this to the discipline-specific variable label conventions or to the projection. At this time the interviewer conveyed the physics convention for the spherical coordinate system and Tyler changed the $\sin \beta$ to $\sin \alpha$. A second attempt at integration still yielded 0 , which Tyler connected to the difference in how $\theta$ and $\alpha$ are defined. However, Tyler still did not connect this to his differential length element or recognize the need for the length vector to have three components. Tyler further drew upon graphical representations of sine and cosine functions to illustrate the change in the angle as a mathematical shift. The rote-computational reasoning resulting in the change in the trigonometric expression substituted for a connection to the projection, as it did for Rachel and Silas.

Notably, this use of mathematical formalism rather than geometric reasoning presents even in the interviews in which students treated the differential as a product of lengths: groups $\mathrm{CD}$ and $\mathrm{EF}$ first see the shift as mathematical transformation before identifying the geometric motivation. Students here engaged with the "doing" of mathematics first and sense-making second. Furthermore, geometric reasoning was not easily accessed, even though the task involved quantities directly related to coordinate system geometry.

\section{CONCLUSIONS}

The construction of and ability to reason about nonCartesian differential length and volume elements are keys to many of the concepts in E\&M that make use of vector calculus. This work shows that students do not necessarily have a strong understanding of the geometrical aspects of three-dimensional polar coordinate systems that are important to the invocation or construction of these differential elements in physics contexts in particular.

Our results suggest that students struggle to think critically about the aspects that go into the construction of differential elements, but that some are able to check the validity of their expressions and make appropriate adjustments when prompted. Following construction of a differential length vector in an unconventional spherical coordinate system, students used one of three approaches to construct a differential volume element: multiplication of length components, determination of the sides of a differential cube, or recall and transliteration from a spherical differential volume element. The group initially using the second approach did not include a trigonometric term and subsequently switched to recall and transliteration after not being able to determine the justification for inclusion of the term. In general, recall and transliteration was used in groups that had greater difficulty with construction of the differential lengths. These groups either had difficulty recognizing the need to account for multiple components, suggesting that the task did not evoke the component and direction aspect of the differential vector concept image, or instead constructed a differential length vector with Cartesian unit vectors. Additionally, these groups did not try to connect the expressions for the differential length vector and differential volume element.

Furthermore, the construction and checking aspects of these tasks provide stark contrast between those groups who could connect the necessary geometric ideas to the differential volume and those who could not. The checking process only cued projection to students who were already performing stronger on the task and had accessed arc length or projection during length construction [18], while others only saw the use of cosine as the result of a variable change from $\theta$ to $\alpha$ into the sine term in the spherical differential volume. Thus some students have an incomplete understanding of the coordinate systems due to misapplication of particular ideas, while for other students the prerequisite ideas are sometimes present but not accessed or activated in this particular context.

Regardless of variations in students' geometric reasoning ability, the differential volume element appeared more accessible to students than the differential length vector, but the disconnect between the differential elements for students made it difficult for students to construct or correct their length elements accordingly. Two groups in particular attempted to use the spherical differential volume element to make sense of their schmerical length vector after failing to directly recall a spherical length vector. Students' difficulty reconstructing a spherical differential length from these terms, as well as an overarching difficulty recognizing the need for a trigonometric projection, further supports earlier work reporting student difficulty accessing necessary aspects for the construction of a differential length vector [18].

Lastly, overreliance on spherical coordinates and attempts to map trigonometric functions directly are findings reminiscent of $x, y$ syndrome [36], in which a particular process is remembered in terms of symbols rather than how it comes about. The symbols and trigonometric functions of the differential volume element are remembered in the way they are first taught and lose particular meaning over continued use. This is consistent with the formation of a restricted concept image [30]: prolonged use of a particular idea in a formulaic context or limited range of situations can obscure underlying understanding. Thus when students meet a broader context, they struggle with the application of fundamental ideas. Bollen and colleagues similarly report that students are able to perform calculations with differential vector operators but struggle to interpret the conceptual meaning [17]. Our students' mostly computational use of spherical volume and area elements earlier in the semester appears to obscure any underlying understanding of how these elements are constructed. Thus when students work within an unconventional system, they struggle to access or apply appropriate concepts. 
The accessibility of the differential volume elements coupled with students' failure to connect mathematical aspects to geometric aspects imply that in order to improve instruction of non-Cartesian differential elements in E\&M, more focus should be given to how length, area, and volume elements are constructed and determined when problem solving, with explicit emphasis on building the requisite ideas by connecting them to geometric aspects and motions within the space of the coordinate systems. In order to address these concerns, results of this study have been used to develop preliminary instructional resources in the style of Tutorials in Introductory Physics [37] to be used at the beginning of E\&M or in a mathematical methods for physics course. These activities structure students' construction of a differential length element in schmerical coordinates in order to engage them with the act of element construction within a non-Cartesian system, and additionally use 3D physical manipulatives to allow students to construct the elements within a physical space in order to elicit geometric reasoning. Based on the pedagogical value of the differential volume construction and checking tasks in helping students recognize issues with the differential length expressions in the interviews, these tasks are included in the materials. Preliminary results of the implementation are promising: the materials seem to generate discussions similar to those in the interviews but allow students to harness an understanding of the physical space, to realize the geometric features of the differential length elements, and to connect those features to properties of the differential volume element. Ongoing testing and development are occurring, and we hope to report on the effectiveness of these materials in the future.

\section{ACKNOWLEDGMENTS}

The authors would like to acknowledge Michael Loverude and Mikayla Mays for their part in organizing aspects of the running of this study. This material is based upon work supported by the National Science Foundation under Grant No. PHY-1405726.
[1] R. Pepper, S. Chasteen, S. Pollock, and K. Perkins, Observations on student difficulties with mathematics in upper-division electricity and magnetism, Phys. Rev. ST Phys. Educ. Res. 8, 010111 (2012).

[2] T. Dray and C. Manogue, The vector calculus gap: Mathematics physics, PRIMUS 9, 21 (1999).

[3] T. Dray and C. A. Manogue, Using differentials to bridge the vector calculus gap, Coll. Math. J. 34, 283 (2003).

[4] T. Dray and C. Manogue, Miscellanea spherical coordinates, Coll. Math. J. 34, 283 (2003).

[5] J. Stewart, Multivaiable Calculus, 8th ed. (Cengage Learning, Boston, MA, 2016).

[6] D. Hu and N.S. Rebello, Understanding student use of differentials in physics integration problems, Phys. Rev. ST Phys. Educ. Res. 9, 020108 (2013).

[7] D. Meredith and K. Marrongelle, How students use mathematical resources in an electrostatics context, Am. J. Phys. 76, 570 (2008).

[8] L. Doughty, E. McLoughlin, and P. van Kampen, What integration cues, and what cues integration in electromagnetism, Am. J. Phys. 82, 1093 (2014).

[9] D. Hu and N. Sanjay Rebello, Using conceptual blending to describe how students use mathematical integrals in physics, Phys. Rev. ST Phys. Educ. Res. 9, 020118 (2013).

[10] D. Nguyen and N. S. Rebello, Students' difficulties with integration in electricity, Phys. Rev. ST Phys. Educ. Res. 7, 010113 (2011).

[11] J. Guisasola, J. Almudi, J. Salinas, K. Zuza, and M. Ceberio, The Gauss and Ampere laws: Different laws but similar difficulties for student learning, Eur. J. Phys. 29, 1005 (2008).
[12] C. Singh, Student understanding of symmetry and Gauss's law of electricity, Am. J. Phys. 74, 923 (2006).

[13] A. L. Traxler, K. E. Black, and J. R. Thompson, Student's use of symmetry with Gauss's law, AIP Conf. Proc. 883, 173 (2007).

[14] C. Manogue, K. Browne, T. Dray, and B. Edwards, Why is Ampère's law so hard? A look at middle-division physics, Am. J. Phys. 74, 344 (2006).

[15] C. Wallace and S. Chasteen, Upper-division students' difficulties with Ampère's law, Phys. Rev. ST Phys. Educ. Res. 6, 020115 (2010).

[16] C. Baily and C. Astolfi, Student reasoning about the divergence of a vector field, Proceedings of the Physics Education Research Conference 2014, Minneapolis, MN, 2014, https://www.compadre.org/Repository/document/ ServeFile.cfm?ID=13440\&DocID=4039.

[17] L. Bollen, P. van Kampen, and M. De Cock, Students' difficulties with vector calculus in electrodynamics, Phys. Rev. ST Phys. Educ. Res. 11, 020129 (2015).

[18] B. P. Schermerhorn and J. R. Thompson, preceding article, Physics students' construction of differential length vectors in an unconventional spherical coordinate system, this issue, Phys. Rev. Phys. Educ. Res. 15, 010111 (2019).

[19] B. Schermerhorn and J. Thompson, Student determination of differential area elements in upper-division physics, in Proceedings of the Physics Education Research Conference 2017, Cincinnati, OH, 2017, https://www .compadre.org/Repository/document/ServeFile.cfm?ID= $14644 \&$ DocID=4821.

[20] L. Bollen, P. Van Kampen, C. Baily, and M. De Cock, Qualitative investigation into students' use of divergence 
and curl in electromagnetism, Phys. Rev. Phys. Educ. Res. 12, 020134 (2016).

[21] B. E. Hinrichs, Writing position vectors in 3-d space: A student difficulty with spherical unit vectors in intermediate E\&M, AIP Conf. Proc. 1289, 173 (2010).

[22] T. Paoletti, K. C. Moore, J. Gammaro, and S. Musgrave, Students' emerging understanding of the polar coordinate system, Proceedings of the 16th Annual Conference on Research in Undergraduate Mathematics Education, Denver, Colorado, edited by S. Brown, G. Karakok, K. H. Roh, and M. Oehrtman (Mathematical Association of America, Washington, DC, 2013).

[23] E. Sayre and M. Wittmann, Plasticity of intermediate mechanics students' coordinate system choice, Phys. Rev. ST Phys. Educ. Res. 4, 020105 (2008).

[24] K. C. Moore, T. Paoletti, and S. Musgrave, Covariational reasoning and invariance among coordinate systems, J. Math. Behav. 32, 461 (2013).

[25] K. C. Moore, T. Paoletti, and S. Musgrave, Complexities in students' construction of the polar coordinate system, J. Math. Behav. 36, 135 (2014).

[26] M. Artigue, J. Menigaux, and L. Viennot, Some aspects of students' conceptions and difficulties about differentials, Eur. J. Phys. 11, 262 (1990).

[27] S. Jones, Understanding the integral: Students' symbolic forms, J. Math. Behav. 32, 122 (2013).

[28] V. Sealey and J. R. Thompson, Students' interpretation and justification of "backward" definite integrals, in Proceedings of the 19th Annual Conference on Research in Undergraduate Mathematics Education, Pittsburgh, Pennsylvania (Mathematical Association of America, Washington, DC, 2013).
[29] J. Wagner, Students' obstacles and resistance to Riemann sum interpretations of the definite integral, in Proceedings of the 19th Annual Conference on Research in Undergraduate Mathematics Education, Pittsburgh, Pennsylvania, edited by T. Fukawa-Connelly, N. Engelke Infante, M. Wawro, and S. Brown (Mathematical Association of America, Washington, DC, 2013).

[30] D. Tall and S. Vinner, Concept image and concept definition in mathematics with particular reference to limits and continuity, Educ. Stud. Math. 12, 151 (1981).

[31] D. Griffiths, Introduction to Electrodynamics, 4th ed. (Pearson Education, New York, 2013).

[32] B. Parzysz, "Knowing" vs "Seeing". Problems of the plane representation of space geometry figures, Educ. Stud. Math. 19, 79 (1988).

[33] B. Parzysz, Representation of space and students' conceptions at high school level, Educ. Stud. Math. 22, 575 (1991).

[34] J. Tuminaro and E. F. Redish, Elements of a cognitive model of physics problem solving: Epistemic games, Phys. Rev. ST Phys. Educ. Res. 3, 020101 (2007).

[35] T. J. Bing and E. F. Redish, Analyzing problem solving using math in physics: Epistemological framing via warrants, Phys. Rev. ST Phys. Educ. Res. 5, 020108 (2009).

[36] P. White and M. Mitchelmore, Conceptual knowledge in introductory calculus, J. Res. Math. Educ. 27, 79 (1996).

[37] L. C. McDermott, P. S. Shaffer and the Physics Education Group, Tutorials in Introductory Physics (Prentice Hall, Englewood Cliffs, NJ, 2002). 